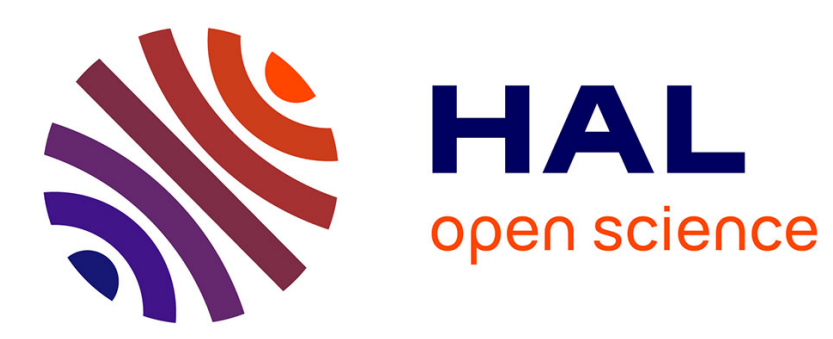

\title{
Modélisation par équations intégrales du frottement sur un demi-espace élasto-plastique
}

\author{
Guy Lederer, Marc Bonnet, H.M. Maitournam
}

\section{To cite this version:}

Guy Lederer, Marc Bonnet, H.M. Maitournam. Modélisation par équations intégrales du frottement sur un demi-espace élasto-plastique. Revue Européenne des Éléments Finis, 1998, 7 (1-3), pp.131-147. 10.1080/12506559.1998.11690470 . hal-00111253

\section{HAL Id: hal-00111253 \\ https://hal.science/hal-00111253}

Submitted on 9 Aug 2008

HAL is a multi-disciplinary open access archive for the deposit and dissemination of scientific research documents, whether they are published or not. The documents may come from teaching and research institutions in France or abroad, or from public or private research centers.
L'archive ouverte pluridisciplinaire HAL, est destinée au dépôt et à la diffusion de documents scientifiques de niveau recherche, publiés ou non, émanant des établissements d'enseignement et de recherche français ou étrangers, des laboratoires publics ou privés. 


\title{
Modélisation par équations intégrales du frottement sur un demi-espace élasto-plastique
}

\author{
Guy Lederer $^{\star, \star \star}$ - Marc Bonnet ${ }^{\star}$ - Habibou M. Maitournam ${ }^{\star}$ \\ * Laboratoire de Mécanique des Solides, CNRS URA 317, Ecole Polytechnique \\ 91128, PALAISEAU cedex (bonnet@athena.polytechnique.fr)

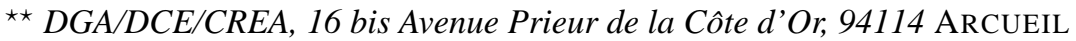

RÉSUMÉ : Cette communication traite d'une modélisation par équations intégrales régularisées du contact sur des massifs élastoplastiques, dans le cadre des déformations planes. Cette formulation, développée ici pour l'indentation et le roulement/glissement continu sur un demiespace homogène isotrope, représente une extension naturelle à des problèmes non-linéaires des méthodes analytiques (élasticité linéaire) de la mécanique du contact classique.

Deux versions (charge fixe ou en mouvement stationnaire) de l'intégration plastique, reposant sur un schéma implicite, ont été mises en æuvre. L'algorithme stationnaire permet de traiter des passages multiples de charge et de calculer des états stabilisés.

Des exemples numériques illustrent les différents aspects (calcul en charge fixe ou mobile, pression de contact donnée a priori ou déterminée itérativement, classification des états stabilisés en fonction du chargement et du coefficient de frottement) de la méthode.

ABSTRACT : This paper deals with a regularized integral equation formulation for contact problems on elastic-plastic bodies, under the plane strain assumption. The formulation has been implemented for indentation and rolling/sliding contact problems on isotropic homogeneous half-planes. It constitutes a natural extension to non-linear problems of the classical analytical methods developed for linear elastic contact mechanics.

Two versions of an implicit elastic-plastic constitutive integration algorithm, dealing with loads respectively fixed or moving at constant velocity, have been implemented in this integral equation framework. The steady-state version can be applied to multiple rolling/sliding loads and to the determination of the stabilised elastic-plastic state.

Several numerical examples illustrate the various aspects of our formulation : fixed or moving load, contact pressure known a priori or iteratively computed, numerical classification of the stabilised elastic-plastic states reached as a function of the load and friction coefficient.

MOTS-CLÉS : Elastoplasticité, équations intégrales, contact, calcul stationnaire, implicite.

KEYWORDS : Elastoplasticity, integral equations, contact, steady-state loading, implicit scheme.

Revue Européenne des Eléments Finis, 7 :131-147 (1998) 


\section{Introduction}

Dans de nombreux cas pratiques (contact roue/rail, roulement/glissement de billes...), le contact de deux solides peut être modélisé par deux demi-espaces en contact suivant une petite portion de leur frontière. Ceci consiste en fait à supposer que l'aire de contact est petite par rapport aux dimensions caractéristiques des solides en contact, et donc que seul un développement du profil local dans la zone de contact potentiel est à prendre en compte pour le calcul des pressions de contact. La mécanique élastique linéaire du contact s'est développée sur cette base, faisant largement appel aux notions d'équations intégrales singulières [Mus53, Joh85]. Cette théorie, limitée à une analyse élastique du contact, ne permet pas la prise en compte de déformations inélastiques.

Le contact élastoplastique est fréquemment traité au moyen de la méthode des éléments finis [BHR85]. Elle présente l'avantage d'une grande généralité mais nécessite, pour les contacts de type ponctuel, un domaine de calcul dont les dimensions sont grandes par rapport à celles de la zone de contact afin que les conditions aux limites extérieures soient sans influence sur la zone de contact.

Les méthodes d'équations intégrales, qui fournissent des solutions élastiques exactes dans le cadre du contact de $i i$ demi-espaces $i i$, permettent en fait de prendre en compte des déformations ii initiales $i \dot{i}$, d'origine plastique et sont donc applicables aux problèmes de contact élastique. Elles sont très bien adaptées à des domaines de calcul infinis (ici : demi-espaces), et conduisent dans le présent contexte à une discrétisation limitée à (i) la portion de surface libre susceptible d'entrer en contact et (ii) la zone potentiellement plastique, bornée, du domaine. Elles fournissent ainsi une extension directe aux cas non-linéaires de la mécanique du contact classique.

Cet article a pour objet de présenter l'approche par équations intégrales pour le contact élastoplastique. Deux algorithmes d'intégration plastique distincts [Ngu77, DVM93] y sont incorporés, permettant de traiter deux types de problèmes : indentation et roulement/glissement continu.

Une des difficultés survenant dans une formulation non-linéaire par équations intégrales est le calcul des contraintes dans les zones potentiellement plastiques. Nous proposons une méthode de régularisation générale ne faisant intervenir aucune hypothèse sur la forme de la solution fondamentale. La démarche présentée dans cet article est ainsi généralisable à des configurations matérielles plus complexes, comme le demi-plan anisotrope ou revêtu.

\section{Formulation par équations intégrales d'un problème d'équilibre avec déformations inélastiques}

Se plaçant dans le cadre de l'hypothèse des petites perturbations, on considère un solide $\Omega$ de frontière $\Gamma$. Notons par $\sigma, \varepsilon, \varepsilon^{p}$ et $\boldsymbol{u}$ les contraintes, déformations, déformations plastiques et déplacements dans $\Omega$, respectivement ; ces grandeurs sont reliées par la loi de comportement élastique $\boldsymbol{\sigma}=\boldsymbol{L}:\left(\varepsilon-\varepsilon^{p}\right)$ et les conditions de compatibilité $\varepsilon=\frac{1}{2}\left(\boldsymbol{\nabla} \boldsymbol{u}+{ }^{T} \boldsymbol{\nabla} \boldsymbol{u}\right)$.

D'autre part, notons par $\boldsymbol{U}^{k}(\boldsymbol{x}, \boldsymbol{y}), \boldsymbol{\Sigma}^{k}(\boldsymbol{x}, \boldsymbol{y})$ une solution fondamentale (déplacement 
et contrainte), c'est-à-dire la réponse élastique en $\boldsymbol{y} \in E$ d'un domaine $E \supset \Omega$ à une force ponctuelle unitaire de direction $\boldsymbol{e}_{k}$ appliquée en un point fixe $\boldsymbol{x} \in \Omega$ arbitrairement choisi. Ces solutions fondamentales ont des expressions analytiques connues si $E$ est l'espace infini entier (solution de Kelvin) ou un demi-espace (solution de Mindlin). Ces tenseurs vérifient dans tous les cas $\boldsymbol{\Sigma}^{k}=\frac{1}{2} \boldsymbol{L}:\left(\boldsymbol{\nabla} \boldsymbol{U}^{k}+{ }^{T} \boldsymbol{\nabla} \boldsymbol{U}^{k}\right)$. On introduit également les vecteurs-contrainte $\boldsymbol{t}=\boldsymbol{\sigma} . \boldsymbol{n}$ et $\boldsymbol{T}^{k}=\boldsymbol{\Sigma}^{k} . \boldsymbol{n}$ associés respectivement à l'état inconnu et à la solution fondamentale ( $\boldsymbol{n}$ : normale extérieure à $\Gamma$ ). Avec ces notations et en l'absence de forces volumiques, tout état élastique sur $\Omega$ avec distribution de déformation $i i$ initiale $i i \varepsilon^{p}$ vérifie l'équation intégrale [Bon95] :

$$
\begin{aligned}
\kappa u_{k}(\mathbf{x})=\int_{\Omega} \varepsilon^{p}(\boldsymbol{y}): \boldsymbol{\Sigma}^{k}(\boldsymbol{x}, \boldsymbol{y}) d V_{y} & \\
& +\int_{\Gamma} \boldsymbol{t}(\boldsymbol{y}) \cdot \boldsymbol{U}^{k}(\boldsymbol{x}, \boldsymbol{y}) d S_{y}-\int_{\Gamma} \boldsymbol{T}^{k}(\boldsymbol{x}, \boldsymbol{y}) \cdot \boldsymbol{u}(\boldsymbol{y}) d S_{y}
\end{aligned}
$$

avec $\kappa=1$ pour $\boldsymbol{x} \in(\Omega-\Gamma), \kappa=0$ pour $\boldsymbol{x} \notin \Omega$; la variable d'intégration est $\boldsymbol{y}$. Pour le cas $\boldsymbol{x} \in \Gamma$, la singularité de $\boldsymbol{U}^{k}$ et $\boldsymbol{T}^{k}$ nécessite dans le cas général une régularisation qui a été largement étudiée [Bon95]. Le tenseur fondamental $\boldsymbol{\Sigma}^{k}$ est singulier en $1 / r^{2}$ en $3 \mathrm{D}(r=\|\boldsymbol{y}-\boldsymbol{x}\|)$ et en $1 / r$ en $2 \mathrm{D}$. L'intégrale de volume du second membre converge donc, mais l'évaluation du gradient des déplacements $\frac{\partial u_{k}}{\partial x_{\ell}}$ pour le calcul des contraintes pose problème.

Bui [Bui78] a montré, pour la solution fondamentale de Kelvin, que $\frac{\partial u_{k}}{\partial x_{\ell}}$ est exprimable sous la forme d'une intégrale de domaine singulière en valeur principale de Cauchy plus un terme complémentaire. Une autre solution, indépendante de la solution fondamentale envisagée, consiste à régulariser l'expression [1]. Considérons l'état élastique auxiliaire suivant défini sur $\Omega$ : dilatation libre (absence de liaisons cinématiques) créé par une déformation initiale uniforme (donc compatible) $\varepsilon^{r}$, qui donne un champ de déplacement associé $\boldsymbol{u}^{r}=\boldsymbol{\varepsilon}^{r} . \boldsymbol{y}$, pour $\boldsymbol{y} \in \Omega$ et un champ de contraintes associé nul. On choisit la valeur particulière $\varepsilon^{r}=\varepsilon^{p}(\boldsymbol{x})$ pour $\varepsilon^{r}$. La représentation intégrale [1] appliquée au déplacement $\boldsymbol{u}^{r}$ s'écrit ainsi, pour $\boldsymbol{x} \in$ $\Omega-\Gamma$ :

$$
u_{k}^{r}(\boldsymbol{x})=\varepsilon^{p}(\boldsymbol{x}): \int_{\Omega} \boldsymbol{\Sigma}^{k}(\boldsymbol{x}, \boldsymbol{y}) d V_{y}-\varepsilon^{p}(\boldsymbol{x}): \int_{\Gamma} \boldsymbol{T}^{k}(\boldsymbol{x}, \boldsymbol{y}) \otimes \boldsymbol{y} d S_{y}
$$

En retranchant l'expression ci-dessus de [1], puis en dérivant le résultat par rapport au point d'observation $\boldsymbol{x}$, on obtient après simplifications et réorganisation des termes la forme régularisée de la représentation intégrale du gradient de déplacement :

$$
\begin{aligned}
\frac{\partial u_{k}}{\partial \boldsymbol{x}}-\varepsilon^{p}(\boldsymbol{x})= & \int_{\Omega}\left(\varepsilon^{p}(\boldsymbol{y})-\varepsilon^{p}(\boldsymbol{x})\right): \frac{\partial}{\partial \boldsymbol{x}} \boldsymbol{\Sigma}^{k}(\boldsymbol{x}, \boldsymbol{y}) d V_{y} \\
& +\int_{\Gamma} \boldsymbol{t}(\boldsymbol{y}) \cdot \frac{\partial}{\partial \boldsymbol{x}} \boldsymbol{U}^{k}(\boldsymbol{x}, \boldsymbol{y}) d S_{y}-\int_{\Gamma} \frac{\partial}{\partial \boldsymbol{x}} \boldsymbol{T}^{k}(\boldsymbol{x}, \boldsymbol{y}) \cdot \boldsymbol{u}(\boldsymbol{y}) d S_{y} \\
& -\boldsymbol{\varepsilon}^{p}(\boldsymbol{x}) \cdot \int_{\Gamma} \boldsymbol{y} \cdot \frac{\partial}{\partial \boldsymbol{x}} \boldsymbol{T}^{k}(\boldsymbol{x}, \boldsymbol{y}) d S_{y}
\end{aligned}
$$


Sous réserve que $\varepsilon^{p}$ vérifie une condition de régularité (continuité au sens de Hölder, i.e. $\left.\boldsymbol{\varepsilon}^{p} \in C^{0, \alpha}\right)$ au voisinage de $\boldsymbol{x}$, l'expression $\left(\varepsilon^{p}(\boldsymbol{y})-\varepsilon^{p}(\boldsymbol{x})\right): \frac{\partial}{\partial \boldsymbol{x}} \boldsymbol{\Sigma}^{k}(\boldsymbol{x}, \boldsymbol{y})$ est singulière en $r^{\alpha-3}$ en $3 \mathrm{D}$ et, ou en $r^{\alpha-2}$ en $2 \mathrm{D}$, et son intégrale est convergente. La relation [3] constitue la base de notre développement.

\section{Application au demi-espace homogène isotrope}

Dans cet article, on s'intéresse spécifiquement au cas où $\Omega$ est le demi-plan $\left\{x_{1} \geq\right.$ $\left.0, x_{2}\right\}$ de frontière $\Gamma=\left\{x_{1}=0\right\}$. Notre développement repose sur l'application de la formule [3] avec la solution fondamentale (Melan) du demi-espace homogène isotrope en déformations planes (obtenue par intégration sur $-\infty \leq x_{3} \leq+\infty$ de la solution tridimensionnelle de Mindlin [Min36]); elle donne l'expression des contraintes et déplacements fondamentaux dans le demi-plan $E=\Omega$, vérifiant la condition de surface libre $\boldsymbol{T}^{k}\left(x_{1}=0, x_{2}, \boldsymbol{y}\right) \equiv 0$.

Les contacts ponctuels considérés ici ont toujours lieu sur $\Gamma$. Ainsi l'intégrale fortement singulière $\int_{\Gamma} \boldsymbol{T}^{k}(\boldsymbol{x}, \boldsymbol{y}) \cdot \boldsymbol{u}(\boldsymbol{y}) d S_{y}$ disparaît de [1]; les points de la droite $x_{1}=0$ peuvent être traités comme des points internes [TB81] à l'aide de [1] avec $\kappa=1$. De plus, comme on a $\boldsymbol{t}=\mathbf{0}$ en dehors de la zone de contact, la discrétisation de la frontière se limite à $\Gamma_{c}$, aire de contact potentiel entre les deux solides.

La régularisation [3] suppose a priori $\Omega$ et $\Gamma$ bornés. Ce n'est pas le cas ici, mais cette difficulté est aisément contournée en remarquant que la régularisation n'est nécessaire que dans la zone bornée $\Omega_{p}$ potentiellement plastique. Considérant l'état auxiliaire $\boldsymbol{u}^{r}$ comme défini sur $\Omega_{p}$, la relation [3] devient donc, après application de la loi de comportement élastique :

$$
\begin{aligned}
\boldsymbol{\sigma}(\boldsymbol{x})= & \int_{\Gamma_{c}} \boldsymbol{t}(\boldsymbol{y}) \cdot \boldsymbol{L}: \frac{\partial}{\partial \boldsymbol{x}} \boldsymbol{U}^{k}(\boldsymbol{x}, \boldsymbol{y}) d S_{y}-\boldsymbol{L}:\left\{\boldsymbol{\varepsilon}^{p}(\boldsymbol{x}) \cdot \int_{\Gamma} \boldsymbol{y} \cdot \frac{\partial}{\partial \boldsymbol{x}} \boldsymbol{T}^{k}(\boldsymbol{x}, \boldsymbol{y}) d S_{y}\right\} \\
& +\boldsymbol{L}:\left\{\int_{\Omega_{p}}\left(\varepsilon^{p}(\boldsymbol{y})-\varepsilon^{p}(\boldsymbol{x})\right): \frac{\partial}{\partial \boldsymbol{x}} \boldsymbol{\Sigma}^{k}(\boldsymbol{x}, \boldsymbol{y}) d V_{y}\right\}
\end{aligned}
$$

ce qu'on note formellement $\boldsymbol{\sigma}(\boldsymbol{x})=\boldsymbol{\sigma}^{c}(\boldsymbol{x})+\boldsymbol{\sigma}^{p}(\boldsymbol{x})$ où $\boldsymbol{\sigma}^{c}$ désigne l'intégrale faisant intervenir les pressions de contact et $\sigma^{p}$ fait intervenir les termes plastiques.

Un intérêt pratique de cette formulation est que la discrétisation se limite à la zone potentiellement plastifiée, évitant ainsi des maillages laborieux. On peut également noter d'un point de vue numérique que si les déformations inélastiques sont connues, la résolution itérative du problème de contact ne fait intervenir que les inconnues sur la frontière en intégrant directement l'effet des déformations plastiques. D'autre part, on remarque que $\boldsymbol{\sigma}^{c}$ fait intervenir le tenseur fondamental solution du problème de Flamant, de sorte que pour une représentation constante ou linéaire par morceaux de la pression sur la zone de contact, les intégrales élémentaires sont connues analytiquement [Joh85]. De plus, si l'effet des déformations plastiques sur les pressions de contact est négligé (hypothèse classique) et pour un contact cylindre-plan, la pression n'est autre que celle de Hertz, et l'expression de $\sigma^{c}$ est analytique [McE49].

Ces remarques montrent que notre formulation, à travers le terme $\sigma^{p}$, étend à des problèmes non-linéaires le traitement analytique du contact en élasticité. 


\section{Intégration plastique et calcul stationnaire}

La mise en œuvre numérique a été effectuée pour des matériaux élastoplastiques isotropes à écrouissage cinématique linéaire vérifiant un critère de Von Mises, soit :

$$
f=\|\boldsymbol{\xi}\|-k \leq 0 \quad \boldsymbol{\xi}=\boldsymbol{s}-C \boldsymbol{\varepsilon}^{p} \quad \boldsymbol{s}=\boldsymbol{\sigma}-(1 / 3) \operatorname{tr}(\boldsymbol{\sigma}) \mathbf{1}
$$

$\left(\|\boldsymbol{\xi}\|=(\boldsymbol{\xi}: \boldsymbol{\xi})^{1 / 2}\right), f$ : fonction de charge, $k$ : limite élastique, $C$ : module d'écrouissage). L'intégration plastique est faite selon une approche implicite, l'histoire du chargement étant discrétisée en pas de temps finis, et pour deux types de situations : (i) contact fixe et (ii) roulement-glissement stationnaire.

La discrétisation consiste en éléments de frontière (découpage de la zone de contact potentiel $\Gamma_{c}$ en segments) et en cellules d'intégration (découpage en cellules rectangulaires de la zone potentiellement plastique $\Omega_{p}$ ). Les déformations plastiques sont supposées constantes par cellule. Il est important de noter que $\Omega_{p}$ est a priori borné dans le cas du contact fixe mais infini dans la direction $x_{2}$ dans le cas stationnaire; on suppose alors que $\varepsilon^{p}\left(x_{1}, \pm \infty\right)$ est fini. Des cellules infinies avec une procédure d'intégration numérique adaptée sont alors employées, en supposant $\varepsilon^{p}$ indépendant de $x_{2}$ au-delà d'une certaine distance à la charge. A noter que, sous ces hypothèses, le calcul par intégration numérique de $\sigma^{p}$ a été validé par comparaison à des formules analytiques [BC94].

Algorithme implicite ClassiQue. Sa convergence est prouvée [Ngu77], et peut être accélérée en intégrant dans la projection implicite la nécessaire discrétisation en temps du problème [ST85]. Ce type d'algorithme est adaptable à une formulation par équations intégrales [BM96].

Connaissant à l'état mécanique à un instant $t_{n}$ (critère de plasticité non violé, champ $\boldsymbol{\sigma}_{n}$ statiquement admissible, champ $\varepsilon_{n}$ cinématiquement admissible), on cherche, pour un incrément de chargement donné, $\varepsilon_{n+1}^{p}$ rendant le problème admissible à l'instant $t_{n+1}$. L'algorithme étant implicite, on impose la satisfaction de toutes les relations de comportement à l'instant $t_{n+1}$, et notamment :

$$
\boldsymbol{n}=\frac{\boldsymbol{\xi}_{n+1}}{\left\|\boldsymbol{\xi}_{n+1}\right\|} \quad f\left(\boldsymbol{\xi}_{n+1}, k\right) \leq 0
$$

Pour ce faire, la déformation plastique $\varepsilon_{n+1}^{p}$ en un point est initialisée à la valeur $\varepsilon_{n}^{p}$, et une approximation élastique des contraintes est calculée à l'aide de [3] (discrétisée) :

$$
\begin{gathered}
\boldsymbol{\nabla} \boldsymbol{u}_{n+1,0}(\boldsymbol{x})-\varepsilon_{n+1,0}^{p}(\boldsymbol{x})=\int_{\Omega}\left(\varepsilon_{n+1,0}^{p}(\boldsymbol{y})-\varepsilon_{n+1,0}^{p}(\boldsymbol{x})\right): \frac{\partial}{\partial \boldsymbol{x}} \boldsymbol{\Sigma}^{k}(\boldsymbol{x}, \boldsymbol{y}) d V_{y} \\
+\int_{\Gamma} \boldsymbol{t}_{n+1}(\boldsymbol{y}) \cdot \frac{\partial}{\partial \boldsymbol{x}} \boldsymbol{U}^{k}(\boldsymbol{x}, \boldsymbol{y}) d S_{y}-\varepsilon_{n+1,0}^{p}(\boldsymbol{x}) . \int_{\Gamma} \boldsymbol{y} \cdot \frac{\partial}{\partial \boldsymbol{x}} \boldsymbol{T}^{k}(\boldsymbol{x}, \boldsymbol{y}) d S_{y}
\end{gathered}
$$

puis une approximation élastique des contraintes par :

$$
\boldsymbol{s}_{n+1}^{E}=\boldsymbol{s}_{n}+2 G \Delta \boldsymbol{e}, \quad \boldsymbol{\xi}_{n+1}^{E}=\boldsymbol{s}_{n+1}-C \boldsymbol{\varepsilon}^{p}=\boldsymbol{\xi}_{n}+2 G \Delta \boldsymbol{e}
$$


( $\boldsymbol{e}$ : déviateur de déformation; $\Delta \boldsymbol{e}=\boldsymbol{e}_{n+1,0}-\boldsymbol{e}_{n}$ ). En supposant que $\boldsymbol{s}_{n+1}^{E}$ viole le critère, on a donc :

$$
\boldsymbol{\xi}_{n+1}=\boldsymbol{\xi}_{n+1}^{E}-(2 G+C) \lambda \boldsymbol{n}
$$

Pour conserver dans cette dernière relation la ii direction $i \iota$ de $\boldsymbol{\xi}_{n+1}^{E}$ donnée par [6], il faut donc que [ST85] :

$$
\boldsymbol{n}=\frac{\boldsymbol{\xi}_{n+1}^{E}}{\left\|\boldsymbol{\xi}_{n+1}^{E}\right\|}
$$

Cette dernière relation, associée à [9], permet d'écrire :

$$
\left\|\boldsymbol{\xi}_{n+1}\right\|=\left\|\boldsymbol{\xi}_{n+1}^{E}\right\|-(2 G+C) \lambda
$$

ce qui, reporté dans $f(\boldsymbol{\xi}, k)=0$, donne l'expression de $\lambda$ :

$$
\lambda=\frac{\left\|\boldsymbol{\xi}_{n+1}^{E}\right\|-k}{2 G+C}
$$

et donne donc $\Delta \varepsilon^{p}=\lambda \boldsymbol{n}$. En résumé, pour un pas $\left[t_{n}, t_{n+1}\right]$, notant $i$ le compteur d'itérations :

1. Initialisation : $i=0, \varepsilon_{n+1, i}^{p}=\varepsilon_{n}^{p}$;

2. Evaluation élastique de $\boldsymbol{\xi}_{n+1, i}^{E}$, relations [7], [8];

3. Si $f\left(\boldsymbol{\xi}_{n+1}^{E}\right) \geq 0$ alors : évaluation de [10], [11], calcul de $\Delta \varepsilon_{i}^{p}$, $i \leftarrow i+1$ et retour à 2 ;

4. Sinon : $\varepsilon_{n+1}^{p}=\varepsilon_{n}^{p}+\Delta \varepsilon^{p}$ et fin.

Algorithme StATIONNAIRE. Il s'agit du cas où le chargement se déplace à une vitesse constante $V \boldsymbol{e}_{2}$ (figure 1. Dans les coordonnées $\left(x_{1}, \hat{x}_{2}=x_{2}-V t\right)$ liées au chargement, le problème est indépendant du temps et ne dépend que de la position $\hat{x}_{2}$ par rapport à la charge; pour tout champ physique $X$, on a ainsi

$$
\frac{d X}{d t}=-V \frac{\partial X}{\partial \hat{x}_{2}}
$$

L'intégration plastique dans le repère mobile consiste à calculer l'incrément de déformation plastique $\Delta \varepsilon^{p}$ lorsque l'on passe d'un point $\boldsymbol{x}$ à un point $\boldsymbol{x}-\Delta \hat{x}_{2} \boldsymbol{e}_{2}$ le long d'une ligne de courant (l'accroissement $-\Delta \hat{x}_{2}$ correspondant à un pas de temps $\left.\Delta t=\Delta \hat{x}_{2} / V\right)$.

Etant donnés des états de contrainte et de déformation plastique initiaux (des valeurs non nulles traduisant l'effet de passages précédents de la charge)

$$
\boldsymbol{\xi}_{0}\left(x_{1}\right)=\left(\boldsymbol{s}_{0}-C \boldsymbol{\varepsilon}_{0}^{p}\right)\left(x_{1},+\infty\right)
$$

L'incrément du déviateur de déformation est défini par

$$
\Delta \boldsymbol{e}=\boldsymbol{e}\left(x_{2}-\Delta x_{2}\right)-\boldsymbol{e}\left(x_{2}\right)=\boldsymbol{e}_{n+1}-\boldsymbol{e}_{n}
$$




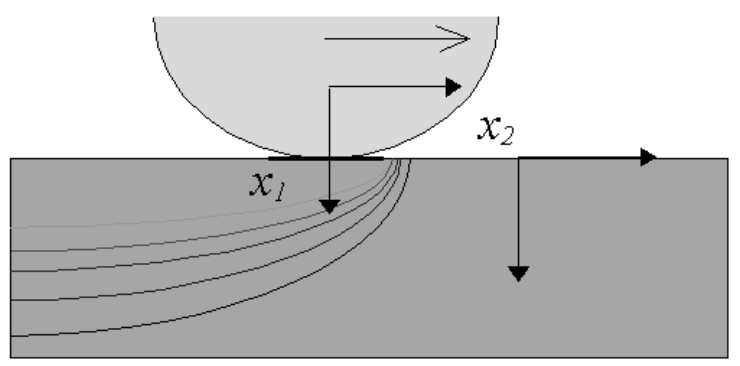

Figure 1: Contact et roulement/glissement stationnaire continu : schéma de principe

Le multiplicateur plastique en fonction de $\boldsymbol{\xi}_{n+1}^{E}$ est encore donné par [11]. Pour un ii passage ii de la charge, l'algorithme, initialement dû à [DVM93], se présente comme suit. Noter que la variable $\hat{x_{2}}$, indicée par $n$, est à la fois spatiale et temporelle, de sorte que l'algorithme produit l'état mécanique dans tout le massif résultant d'un nouveau passage de charge et opère ii globalement $i \dot{i}$, et non point par point.

(A) Initialisation : $i=0$;

(B) Boucle sur $n, m$ :

1. $\boldsymbol{\xi}_{(m, n), i}^{E}=\boldsymbol{\xi}_{0}(m)$;

2. Evaluation élastique de $\boldsymbol{\xi}_{(m, n), i}^{E}$, relations [7] et [8];

Aller en (D)

3. Si $f\left(\boldsymbol{\xi}_{(m, n), i}^{E}\right)>0$, aller en (C)

(C) Projection stationnaire : Boucle sur $n$ :

Boucle sur $m$ :

1. Evaluation de $\boldsymbol{\xi}_{(m . n+1), i+1}^{E}=\boldsymbol{s}_{(m, n), i+1}+2 G\left(\boldsymbol{e}_{(m, n+1), i}-\boldsymbol{e}_{(m, n), i}\right) ;$

2. Si $f\left(\boldsymbol{\xi}_{(m, n+1), i+1}^{E}\right)>0$, alors : projection à l'aide de [10], [11] et $\varepsilon_{(m, n+1), i+1}^{p}=\varepsilon_{(m, n), i+1}^{p}+\Delta \varepsilon^{p}$ sinon : $\varepsilon_{(m, n+1), i+1}^{p}=\varepsilon_{(m, n), i+1}^{p} ;$

$i \leftarrow i+1$ et retour à $(\mathrm{B})$;

(D) Fin

\section{Exemples numériques}

Dans tous les exemples présentés, le matériau a pour caractéristiques $E=210000$ $\mathrm{MPa}, \nu=0,3, k=159 \mathrm{MPa}, C=6900 \mathrm{MPa}$. 


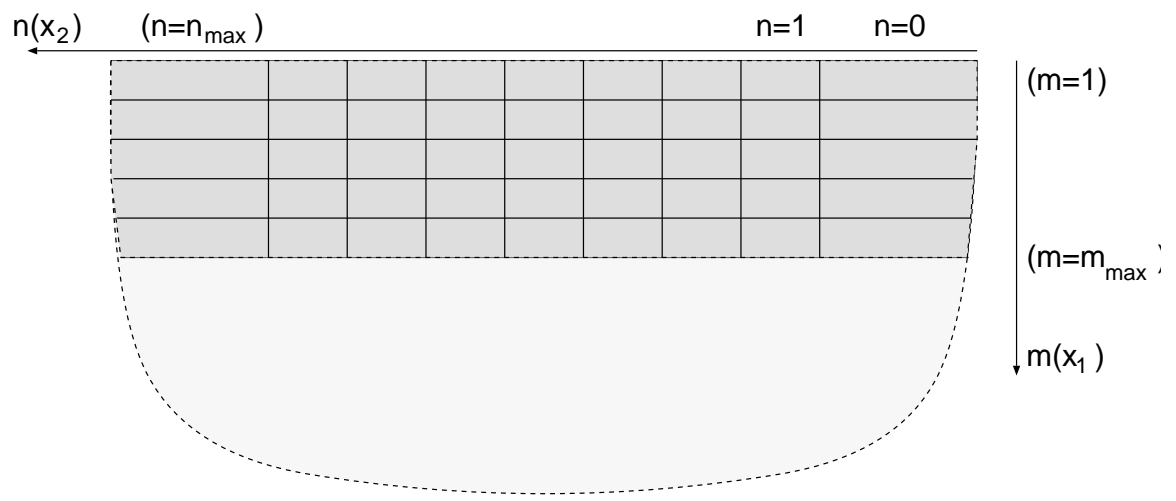

Figure 2: Discrétisation en cellules d'intégration (les cellules pour $n=0$ et $n=n_{\max }$ sont des éléments infinis avec déformation plastique constante

\subsection{Exemples en chargement fixe}

On considère l'indentation d'un massif semi-infini, les pressions de contact étant ici données a priori (répartitions constante ou hertzienne, toutes deux permettant une intégration analytique de $\sigma^{c}$ ).La zone de contact est $\Gamma_{c}=\left\{-\ell_{c} \leq x_{2} \leq \ell_{c}\right\}$ avec ici $\ell_{c}=10 \mathrm{~mm}$.

La figure 3 présente l'évolution suivant la profondeur des déformations plastiques équivalentes sous le centre de contact, pour un chargement constant sur $\Gamma_{c}:\left(t_{1}, t_{2}\right)=$ $(4,4 k ; 0)$; les résultats de notre méthode sont comparés à ceux obtenus par un calcul par éléments finis (code CASTEM 2000).

La figure 4 présente un exemple d'isovaleurs de déformations plastiques sous une charge hertzienne $\left(\ell_{c}=10 \mathrm{~mm}\right.$, pression maximum $\left.p_{0}=8 k\right)$. La zone potentiellement plastique est découpée en $40 \times 40$ cellules; aucune autre discrétisation n'étant nécessaire ici. Par comparaison, la résolution par éléments finis nécessite la discrétisation d'un domaine de calcul au moins sept fois plus grand suivant chaque direction pour stabiliser les grandeurs sous le contact.

\subsection{Exemples en chargement stationnaire}

On considère maintenant le roulement sans glissement : pressions de contact ici encore données a priori $; \ell_{c}=10 \mathrm{~mm}$.

RÉPARTITION HERTZIENNE, $p_{0}=3.8 k$. Il s'agit d'une sollicitation à la limite d'un comportement d'adaptation. Les figures 5 et 6 présentent le champ de déformation plastique équivalente, respectivement pour un premier passage de la charge et en régime stabilisé. La discrétisation de $\Omega_{p}$ est dans ce cas de $121 \times 40$ cellules, la prise en compte des déformations plastiques en amont de la zone maillée se faisant à l'aide 
d'éléments infinis suivant $x_{2}$. On voit clairement apparaitre le caractère stationnaire du champ de déformation plastique. La figure 7 montre la bonne concordance entre nos résultats et ceux issus d'un calcul stationnaire par éléments finis [DVM93].

RÉPARTITION ii CHAPEAU $i i$. La solution élastique est, pour ce cas aussi, connue analytiquement. La discrétisation de $\Omega_{p}$ est dans ce cas de $161 \times 50$ cellules, la prise en compte des déformations plastiques en amont de la zone maillée se faisant à l'aide d'éléments infinis suivant $x_{2}$.

La figure 8 présente le champ de déformation plastique équivalente en régime stabilisé, dans le cas $p_{0}=4,4 k ;$ l'état stabilisé est adapté ( $\varepsilon^{p}$ est indépendant de $\hat{x}_{2}$ ). La figure 9 donne les valeurs de $\varepsilon_{11}^{p}, \varepsilon_{12}^{p}, \varepsilon_{22}^{p}$ en fonction de la profondeur $x_{1}$. Les champs de contrainte $\sigma_{11}$ et $\sigma_{e q}$ (contrainte équivalente de Von Mises) sont représentés sur les figures 10 et 11 respectivement.

La figure 12 donne les états stabilisés atteints en fonction du coefficient de frottement $\mu$ et de la pression $p_{0}$ au centre. Elle est créée par balayage du plan $\left(\mu, p_{0}\right)$, un calcul stationnaire complet étant fait pour chaque couple $\left(\mu, p_{0}\right)$ considéré. Les courbes délimitent les régions du plan $\left(\mu, p_{0}\right)$ conduisant à des états stabilisés purement élastique, adapté ou accommodé (le dédoublement de chaque courbe résulte du pas fini de balayage numérique; par exemple, dans le fuseau inférieur, la courbe inférieure (resp. supérieure) donne pour chaque $\mu$ la valeur de $p_{0}$ la plus élevée (resp. la plus basse) ayant conduit à un état stabilisé élastique (resp. adapté)).

\subsection{Exemple en chargement stationnaire avec contact : influence de la rugosité}

On considère maintenant un chargement glissant exercé par un poinçon de forme ii ondulée $i \measuredangle$ (figure 13). Le contact est supposé sans frottement $(\mu=0)$; les efforts et l'aire de contact sont cette fois déterminés itérativement et numériquement, la portion de surface libre au voisinage de la charge étant découpée en éléments de frontière.

Les figures 14 et 15 montrent respectivement la répartition de pression de contact sous le poinçon ondulé pour les limites élastique et d'adaptation et le champ de contrainte équivalente de von Mises (ondulation d'amplitude 0,007 millimètres). La répartition de contrainte est totalement modifiée par rapport au cas hertzien, et il apparaît notamment une interaction importante entre les trois ii pics $i i$ de pression. On ne peut donc pas se contenter de considérer le contact hertzien entre un demi-plan et un solide ayant un rayon de courbure correspondant à celui de l'ondulation. Ce type de raisonnement conduirait en effet à ne considérer que la pression maximale au centre de contact en négligeant ainsi la redistribution de contraintes mise en évidence par la figure 15. 


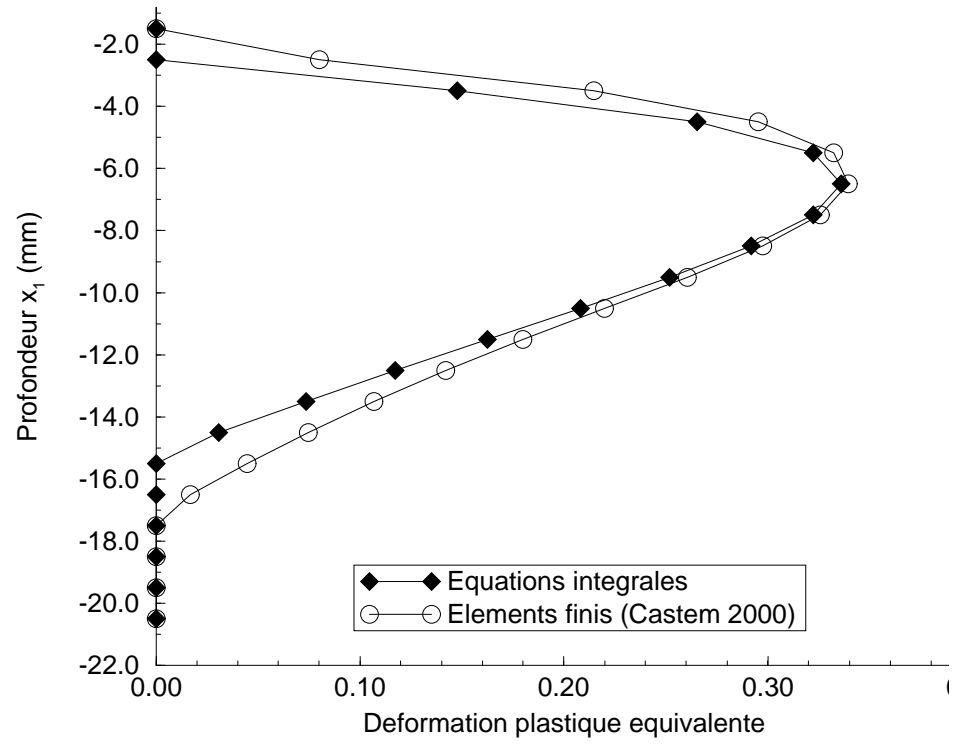

Figure 3: Demi-plan sous pression constante $\left(p_{0}=4,4 k\right)$ : déformation plastique équivalente sous le centre de contact, en fonction de la profondeur $x_{1}$

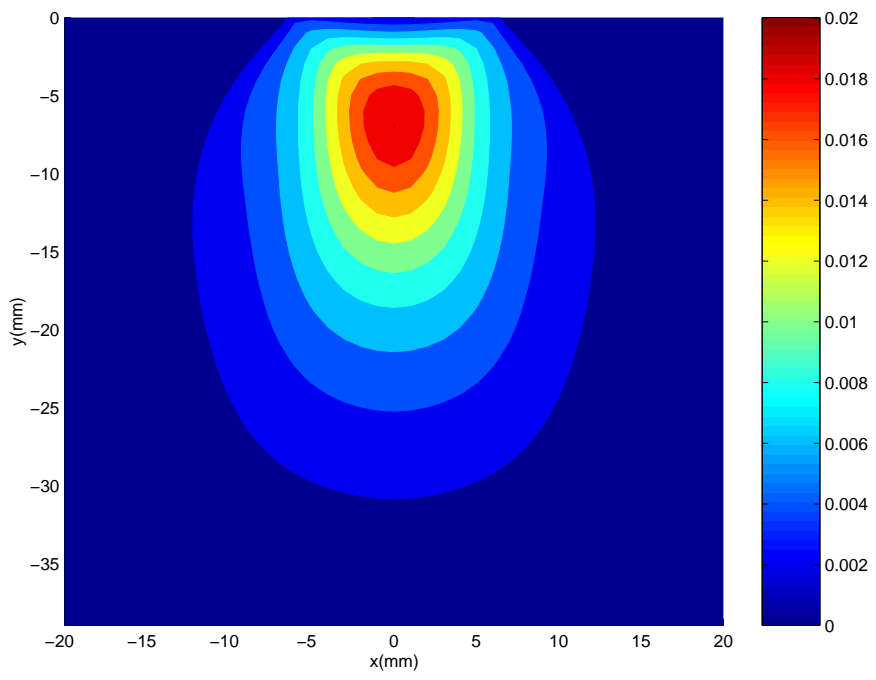

Figure 4: Demi-plan sous pression hertzienne $\left(p_{0}=8 k\right)$ : déformation plastique équivalente 


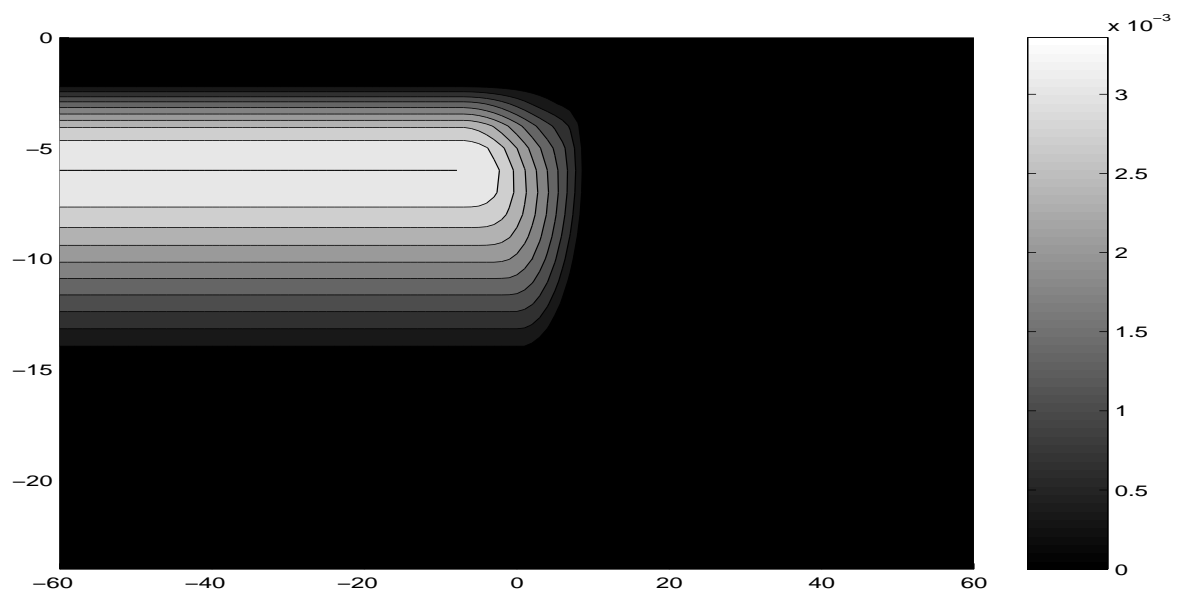

Figure 5: Demi-plan sous pression hertzienne glissante $\left(p_{0}=3,8 k\right)$ : déformation plastique équivalente après le premier passage

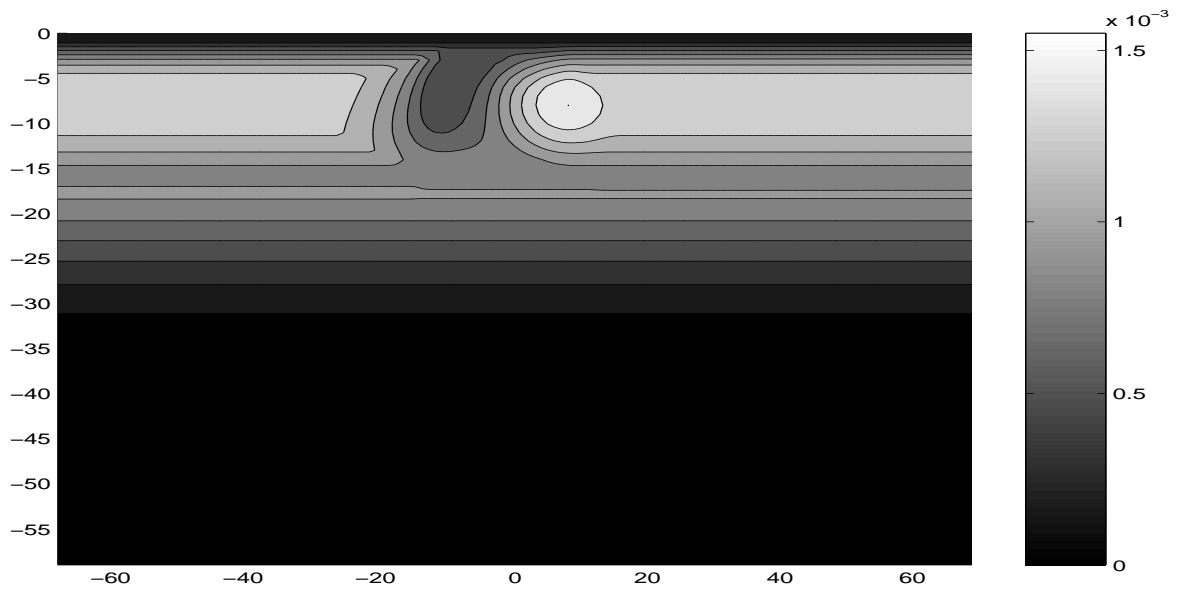

Figure 6: Demi-plan sous pression hertzienne glissante $\left(p_{0}=3,8 k\right)$ : déformation plastique équivalente dans l'état stabilisé 


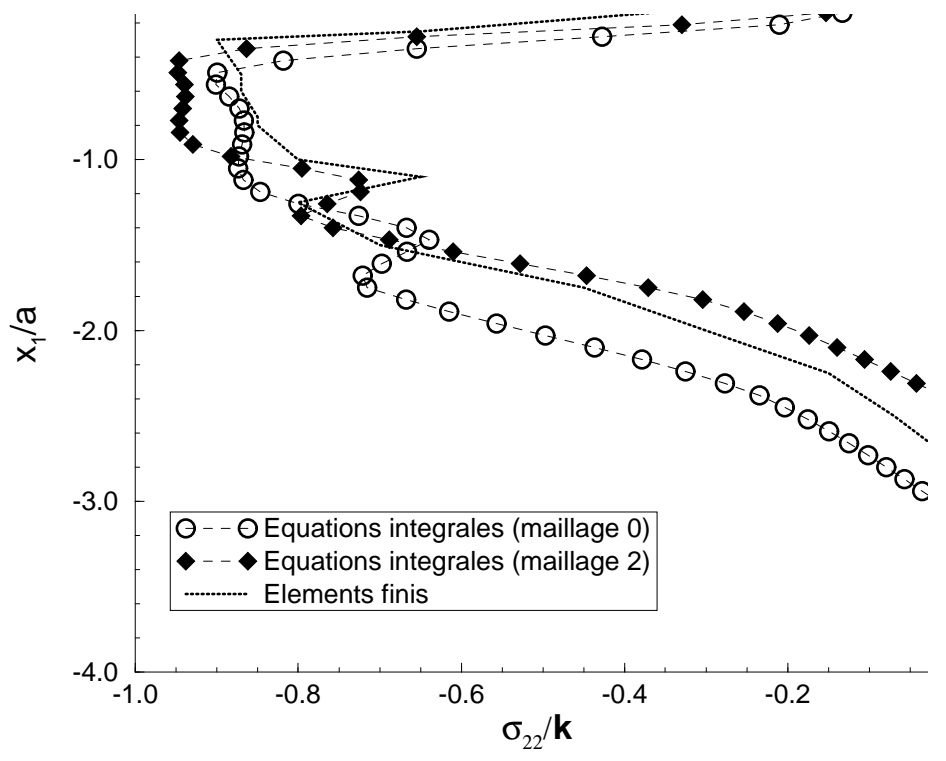

Figure 7: Demi-plan sous pression hertzienne glissante $\left(p_{0}=3,8 k\right)$ : contrainte résiduelle de roulement $\sigma_{22}$ sous le centre de contact; comparaison entre équations intégrales et éléments finis

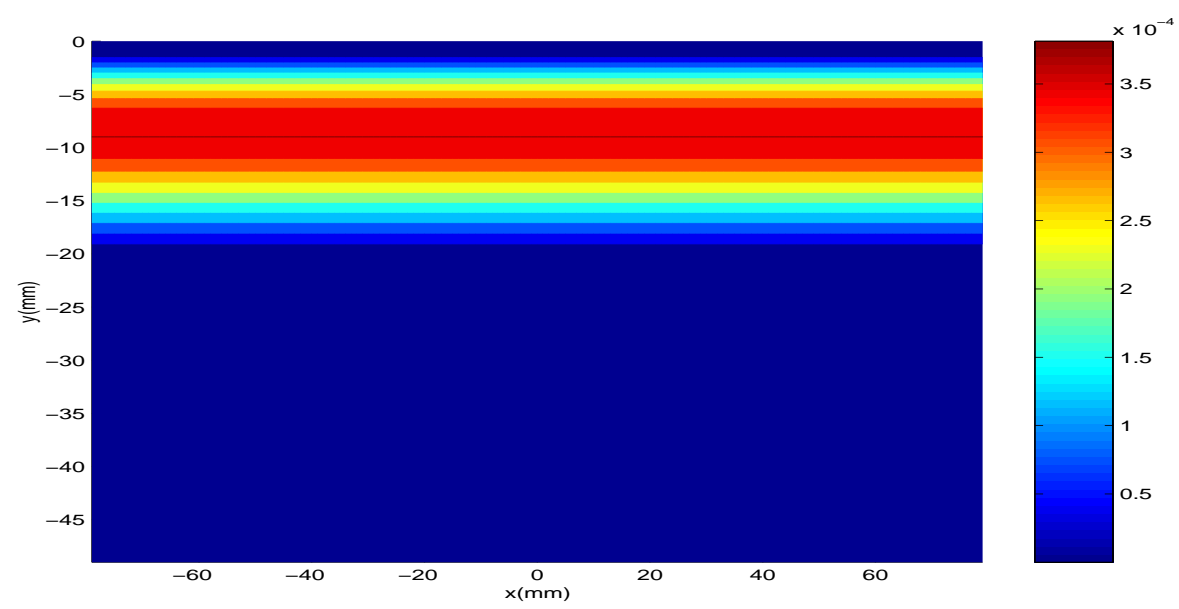

Figure 8: Demi-plan sous pression ii chapeau ¿¿ glissante $\left(p_{0}=4,4 k, \mu=0\right)$ : champ de déformation plastique équivalente 


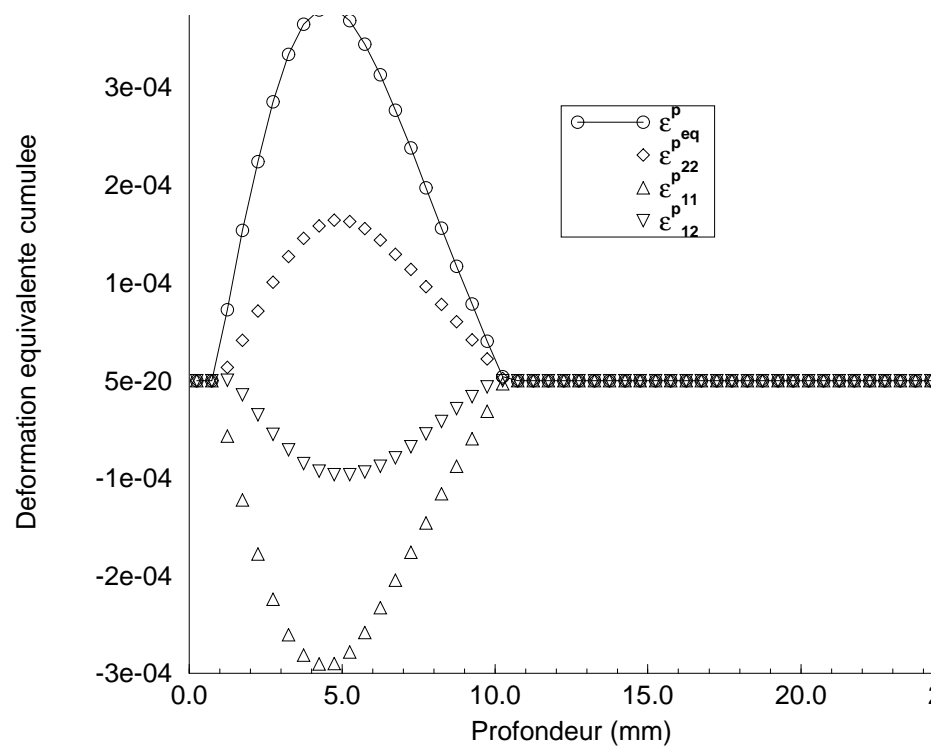

Figure 9: Demi-plan sous pression ii chapeau ¿¿ glissante $\left(p_{0}=3 k, \mu=0\right)$ : déformations plastiques en fonction de la profondeur

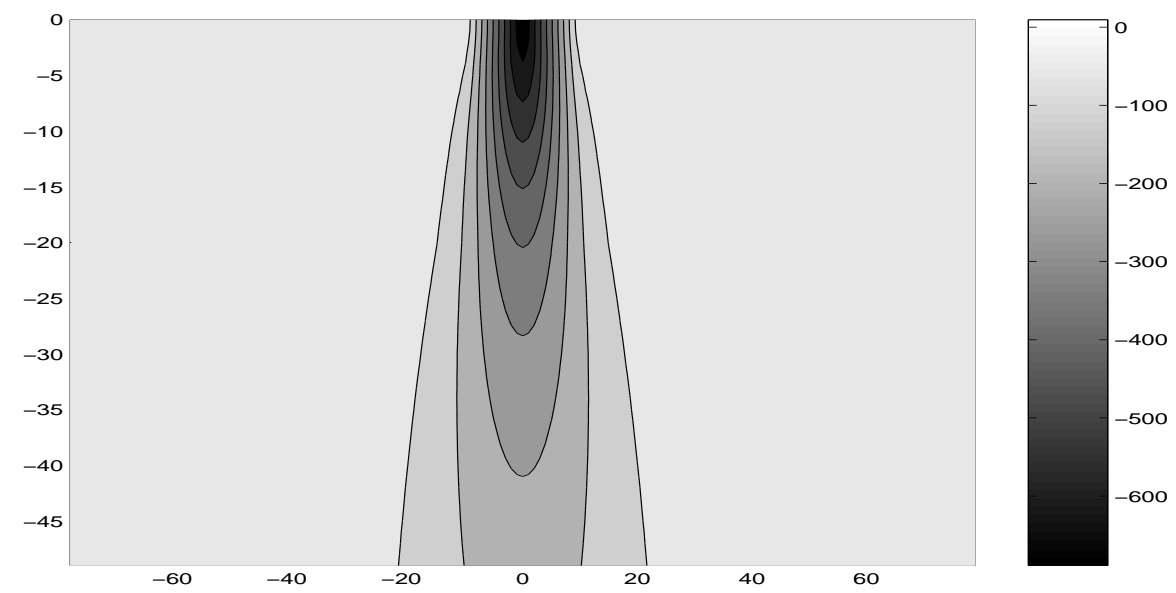

Figure 10: Demi-plan sous pression ii chapeau ¿¿ glissante $\left(p_{0}=3 k, \mu=0\right)$ : champ de contrainte $\sigma_{11}$ 


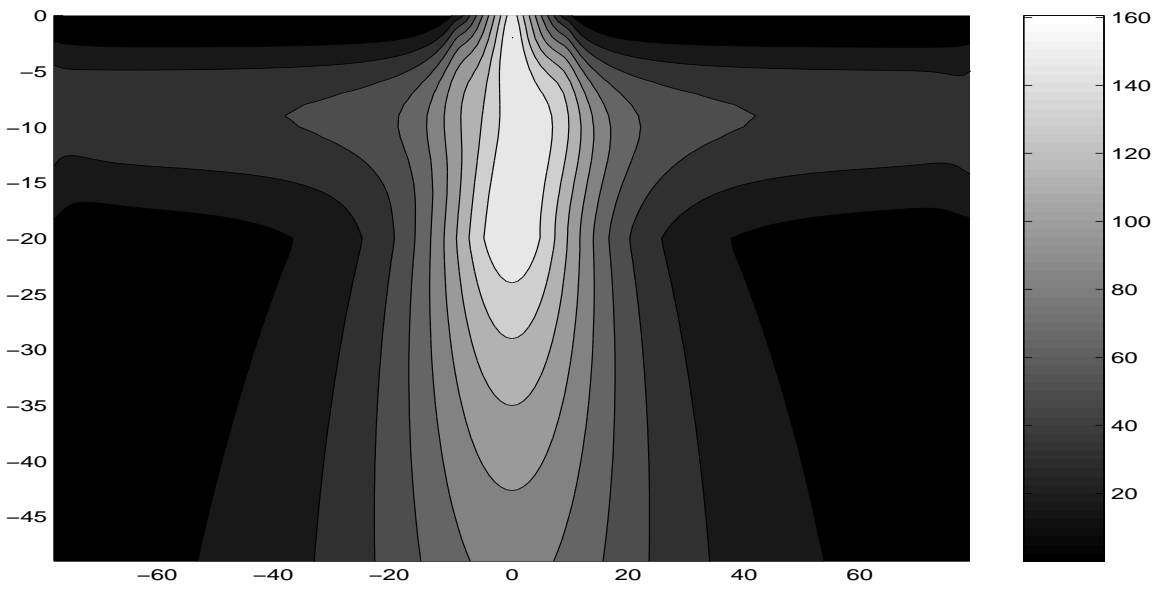

Figure 11: Demi-plan sous pression ii chapeau ¿¿ glissante $\left(p_{0}=3 k, \mu=0\right)$ : champ de contrainte équivalente de Von Mises

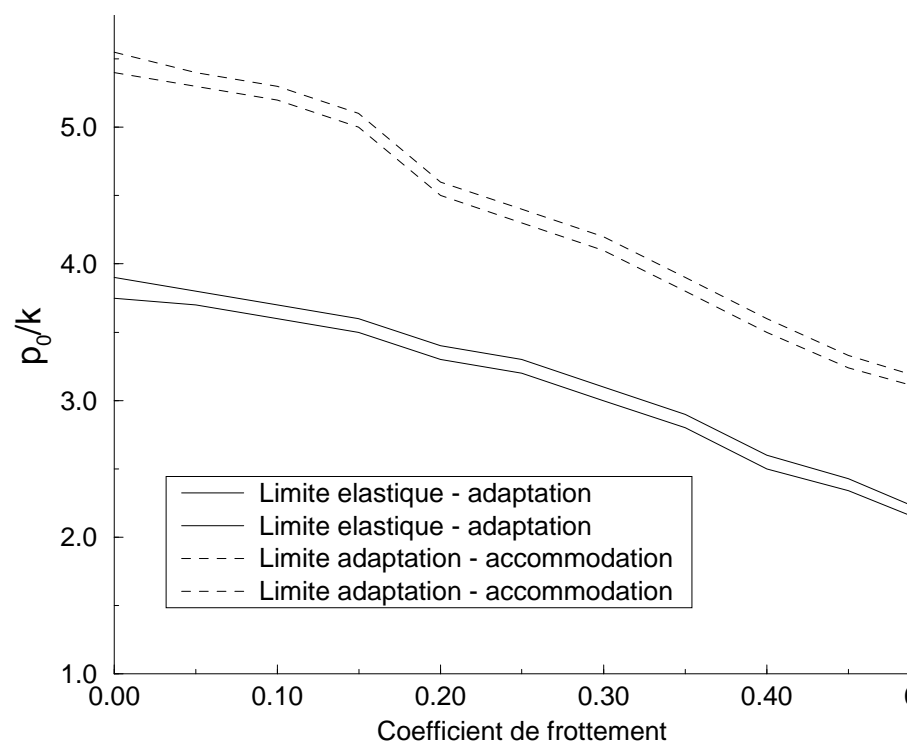

Figure 12: Demi-plan sous pression ii chapeau ¿¿ glissante : régimes stabilisés atteints en fonction de $p_{0}, \mu$ 


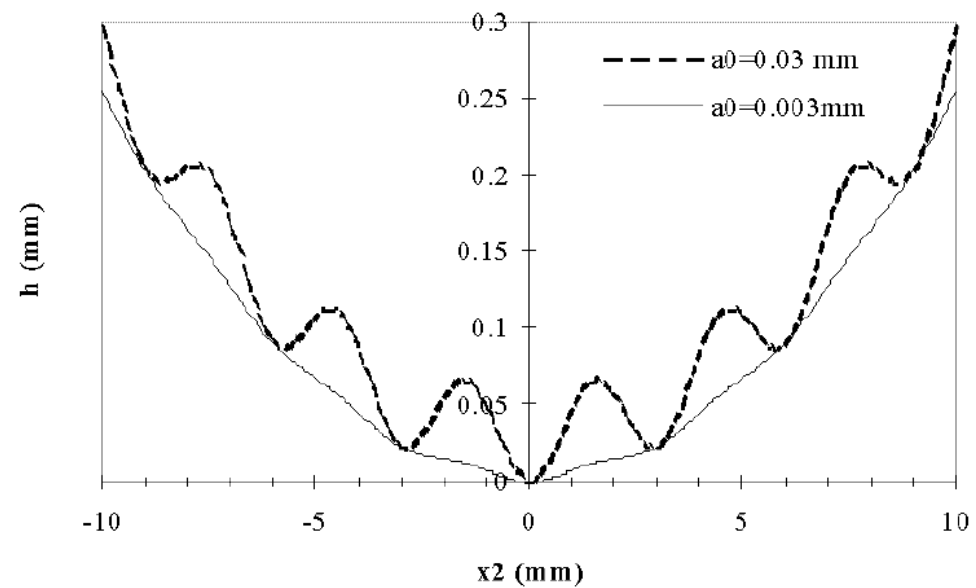

Figure 13: Contact et roulement/glissement stationnaire continu : profil non déformé d'un cylindre avec ondulation, pour deux amplitudes $a_{0}=0,003 \mathrm{~mm}$ et $a_{0}=0,03 \mathrm{~mm}$

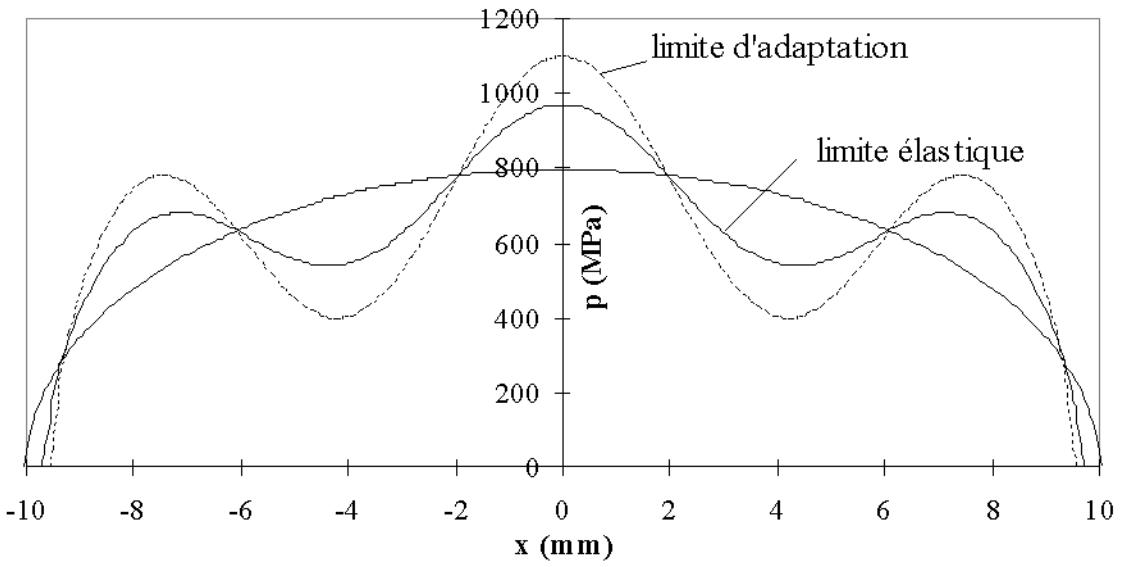

Figure 14: Contact et roulement/glissement stationnaire continu : répartition de pression correspondant à la limite élastique $\left(a_{0}=0,004 \mathrm{~mm}\right)$ et à la limite d'adaptation $\left(a_{0}=0,007 \mathrm{~mm}\right)$ pour un contact ondulé sans frottement 


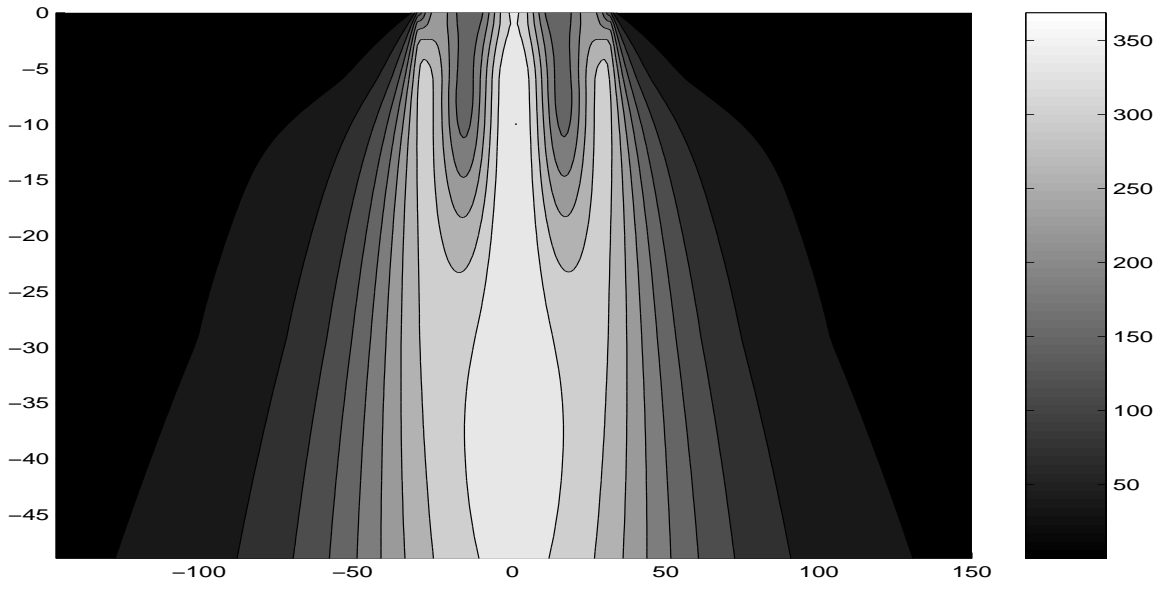

Figure 15: Contrainte de Von Mises équivalente en régime stabilisé (répartition ii ondulée ¿¿ de pression

\section{Conclusion}

La formulation présentée généralise à des problèmes non-linéaires l'approche classique de la mécanique élastique du contact. En particulier, la méthode d'équations intégrales pour le calcul élastoplastique stationnaire proposée ici est originale, et les premiers résultats numériques sont encourageants.

Notre approche est particulièrement adaptée aux calculs en milieu semi-infini, cette caractéristique constituant sans doute l'apport le plus important sur le plan pratique. De nombreuses possibilités d'extensions existent : prise en compte d'un revêtement (couche mince parallèle à la surface libre, de caractéristiques mécaniques distinctes), la solution fondamentale utilisée étant soit celle du demi-espace homogène, soit celle adaptée au matériau bicouche [DH61, HD62] (la première possibilité, la plus simple à mettre en œuvre, permet de prendre en compte une anisotropie éventuelle de l'écart de constantes élastiques); pression de contact initialement inconnue ; roulement et glissement sur un demi-espace (problème tridimensionnel), sur une bande de profondeur finie...

\section{Remerciements}

K. DANG VAN a été pour beaucoup dans le démarrage de ce travail. Pour cela et de nombreux échanges et discussions, nous tenons à le remercier chaleureusement.

La thèse de G. LEDERER [Led97], dont ce travail fait partie, a été soutenue financièrement par une bourse DGA/CNRS. 


\section{Références}

[BC94] Ballard P., Constantinescu A. On the inversion of subsurface residual stresses from surface stress measurements. J. Mech. Phys. Solids, 42, 1767-1788, 1994.

[BHR85] Bhargava V., Hahn G.T., Rubin C. An elastic-plastic finite element model of rolling contact. Part I : analysis of single contacts. ASME J. Appl. Mech., 52, 67-74, 1985.

[BM96] BONNET M., MUKHERJEE S. Implicit BEM formulations for usual and sensitivity problems in elasto-plasticity using the consistent tangent operator concept. Int. J. Solids Struct., 33, 4461-4480, 1996.

[Bon95] Bonnet M. Equations intégrales et éléments de frontière. CNRS Editions / Eyrolles, Paris, France, 1995.

[Bui78] BUI H.D. Some remarks about the formulation of three-dimensional thermoelastoplastic problems by integral equations. Int. J. Solids Struct., 14, 935-939, 1978.

[DH61] Dundurs J., Hetenyi M. The elastic plane with a circular insert, loaded by a radial force. ASME J. Appl. Mech., 28, 103-110, 1961.

[DVM93] Dang VAn K., Maitournam M.H. Steady-state flow in classical elastoplasticity : applications to repeated rolling and sliding contact. J. Mech. Phys. Solids, 41, 1691-1710, 1993.

[HD62] Hetenyi M., Dundurs J. The elastic plane with a circular insert, loaded by a radial force. ASME J. Appl. Mech., 29, 362-368, 1962.

[Joh85] Johnson K.L. Contact mechanics. Cambridge University Press, 1985.

[Led97] LEDERER G. Modélisation tribo-mécanique du frottement en milieu agressif. Thèse de doctorat, Ecole Polytechnique, Palaiseau, France, 1998.

[McE49] MCEwen E. Stress in elastic cylinders in contact along a generatrix. Phil. Mag., 40, 454-460, 1949.

[Min36] Mindlin R. Force at a point in the interior of a semi-infinite solid. Physics, 7, 195-201, 1936.

[Mus53] Mushrelishvili N.I. Some basic problems of the mathematical theory of elasticity. Noordhoff, 1953.

[Ngu77] NGUYEN Q.S. On the elastic-plastic initial-boundary value problem and its numerical integration. Int. J. Num. Meth. in Eng., 11, 817-832, 1977.

[ST85] SIMO J.C., TAYLOR R.L. Consistent tangent operators for rate-independent elastoplasticity. Comp. Meth. in Appl. Mech. Engng., 48, 101-118, 1985.

[TB81] TELles J.C.F., BREBBiA C.A. Boundry element solution for half-plane problems. Int. J. Solids Struct., 17, 1149-1158, 1981. 